\title{
Collective effects in $\nu \bar{\nu}$ synchrotron radiation from neutron stars.
}

\author{
L. B. Leinson \\ Institute of Terrestrial Magnetism, Ionosphere and Radio Wave Propagation \\ RAS, 142092 Troitsk, Moscow Region, Russia
}

\author{
A. Pérez \\ Departamento de Física Teórica, Universidad de Valencia \\ 46100 Burjassot (Valencia), Spain
}

\begin{abstract}
We have considered collective effects in $\nu \bar{\nu}$ synchrotron radiation from an ultrarelativistic degenerate electron gas in neutron stars with strong magnetic fields. For this problem we apply a calculation method which explicitly makes use of the fact that the radiating electron moves semi-classically, but takes into account the interaction among particles in a quantum way. First we apply this method to calculate $\nu \bar{\nu}$ synchrotron radiation by an ultrarelativistic electron in vacuum and we compare this result with that obtained previously by other techniques. When a degenerate plasma is considered, we show that collective effects lead to an essential enhancement (about three times) of the vector weak-current contribution to neutrino pair emissivity.
\end{abstract}

97.60.Jd,95.30.Cq,13.15.-f,52.25.Tx

Typeset using REVTEX 


\section{INTRODUCTION}

Synchrotron radiation of neutrino pairs from a strongly magnetized degenerate gas of ultrarelativistic electrons in a neutron star has been studied by many authors (See [1]- [7]). All cited considerations of the problem are based on a single-particle approach, i.e. except for the Pauli principle, no interaction among the electrons is taken into account. However, due to the electromagnetic interaction, an electron moving along a circular orbit involves in its motion a number of neighboring electrons. In other words, the electron, moving in the electron gas, is followed by a cloud of virtual particles and holes. When annihilating, they also produce a flux of neutrino pairs going in the same direction as $\nu \bar{\nu}$ radiation of the initial electron. To evaluate this effect at the lowest order of coupling constants one has to include in the matrix element of weak interactions two Feynman diagrams, shown in Fig. 1.

By taking into account the Pauli principle, the first diagram describes the mechanism of $\nu \bar{\nu}$ synchrotron radiation discussed in the literature. The second diagram corresponds to the interaction of the initial electron with a weak neutrino field via intermediate particle and hole excitations, which are also included in the photon propagator. Naively, the second diagram contribution to the matrix element might appear to be $e^{2}=1 / 137$ times smaller than the first one. However, as we will show, this is not the case. In fact, the fine structure constant enters only in the medium polarization function $\Pi(\omega, \mathbf{k})$, shown in the diagram as a loop. So, the effective weak interaction via the medium polarization introduces, as compared to the first diagram, an extra factor which, in the case of a transversal virtual photon, is of the order $\Pi /\left(q^{2}-\Pi\right)$, where $q=(\omega, \mathbf{k})$ is the total four-momentum carried out by the neutrino pair. To estimate this factor one should take into account that synchrotron radiation from an ultrarelativistic electron goes into a narrow cone with angle $\theta \sim m / E$ relative to its velocity. Here $m$ and $E$ are the mass and the energy, respectively, of the electron. By this reason one has (we set $\hbar=c=k_{B}=1$ )

$$
q^{2} \lesssim \frac{m^{2}}{E^{2}} \omega^{2} \ll \omega^{2}
$$

i.e., the total four momentum transfer is much smaller than the total energy of the radiated 
neutrino pair. Let us consider a degenerate electron gas under the following condition \&:

$$
\frac{e B}{T E_{F}} \ll 1
$$

Since $\omega$ is of the order of the medium temperature $T$, one has $\omega \gg \omega_{0}$, where $\omega_{0}=e B / E_{F}$ is the Larmor frequency of degenerate electrons near the Fermi energy $E_{F}$. Then one can neglect the small contribution of the magnetic field to the medium polarization, and use the polarization function $\Pi$ of an isotropic electron gas which, when $\omega \rightarrow k$, can be estimated as the square of the plasma frequency, defined as

$$
\omega_{p}^{2}=\frac{4}{3 \pi} e^{2} E_{F}^{2}
$$

Thus, one obtains

$$
\left|\frac{\Pi}{q^{2}-\Pi}\right| \sim\left|\frac{\omega_{p}^{2}}{q^{2}-\omega_{p}^{2}}\right| \sim 1
$$

because

$$
\frac{q^{2}}{\omega_{p}^{2}} \sim \frac{1}{e^{2}} \frac{m^{2}}{E_{F}^{2}} \frac{T^{2}}{E_{F}^{2}}
$$

and the last ratio is small for a degenerate ultrarelativistic electron gas.

Published calculations of $\nu \bar{\nu}$ emissivity due to synchrotron radiation from an electron gas in the neutron star made use of exact wave functions of the electron in a uniform magnetic field. In this case, integration of the squared matrix element leads to either Laguerre or Bessel functions with a complicated behavior, and summation over all initial and final Landau states is a delicate problem. Actually, however, magnetic fields inside neutron stars typically satisfy the condition $\omega_{0} \ll E_{F}$, which means that electrons near the Fermi surface move semi-classically, and there is no reason to use exact Landau states in order to calculate

\footnotetext{
${ }^{1}$ Note that in the opposite case $T \ll \omega_{0}$, the population of excited Landau levels above the Fermi energy exponentially tends to zero, and the synchrotron radiation becomes not relevant as a mechanism of neutrino pair emission.
} 
the neutrino pair emissivity. In order to study $\nu \bar{\nu}$ synchrotron radiation of ultrarelativistic electrons, we apply an alternative method of calculation, which explicitly makes use of the fact that the radiating particle moves semi-classically, although it takes into account the interaction among particles in a quantum way. This method has been developed by Baier and Katkov [8] to consider $\gamma$-synchrotron radiation in vacuum. A detailed derivation of this method applied to $\gamma$-radiation is given, for instance, in [9].

This paper is organized as follows. In order to test our calculation technic, in Section II we focus on $\nu \bar{\nu}$ synchrotron radiation of ultrarelativistic electrons in strongly magnetized vacuum, i.e. we do not set any limitation on the radiated energy of the neutrino pair. We show that the total rate of $\nu \bar{\nu}$ decay, obtained by our calculation technic, coincides with that obtained by other calculation methods [10]- [12]. We also derive a simple formula for the differential rate of decay in vacuum. When multiplied by the appropriate statistical factors,

thus taking into account the Pauli principle, this formula can also be used for the case of an ultrarelativistic electron gas in order to evaluate the contribution of the first diagram shown in Fig. 1, as it is made in Section III. In Section IV we discuss some modifications to be made in our calculation in order to take into account the second diagram shown in Fig.1. We consider the medium polarization tensor, as well as the photon propagator to be used for an ultrarelativistic degenerate electron plasma. In Section V we calculate the contribution of collective effects to the differential rate of neutrino pair emission. By adding these contributions to that obtained in Section III we arrive to a very simple analytic expression for the differential rate of $\nu \bar{\nu}$ synchrotron radiation under the physical conditions we considered. In Section VI we apply this formula to evaluate the energy loss and compare our result with that obtained without taking into account collective effects. We end in Section VII with some discussion and conclusions.

\section{II. $\nu \bar{\nu}$ SYNCHROTRON RADIATION BY ELECTRONS IN VACUUM}


An ultra-relativistic particle radiates into a narrow cone with angle $\theta \sim m / E$ relative to its velocity. By this reason, in the process under consideration, the transferred fourmomentum is much smaller than the $Z$-boson mass

$$
q^{2}=\omega^{2}-\mathbf{k}^{2} \sim \frac{\omega^{2}}{\gamma^{2}}<m^{2} \ll M_{Z}^{2}
$$

Therefore one can use an effective current-current interaction :

$$
\mathcal{L}=\frac{G_{F}}{\sqrt{2}} \bar{\nu} \gamma_{\mu}\left(1-\gamma_{5}\right) \nu \bar{\psi} \gamma^{\mu}\left(C_{V}-C_{A} \gamma_{5}\right) \psi
$$

where $\nu$ represents the neutrino field and $\psi$ stands for the radiating particle field. The coefficients $C_{V}$ and $C_{A}$ are the vector and axial-vector weak coupling constants, respectively, of the radiating particle, which also depend on the neutrino type. In the case of radiating electrons, the combinations that arise, by summing over all neutrino species, are

$$
\begin{gathered}
\sum_{\nu} C_{V}^{2}=\frac{3}{4}-2 \sin ^{2} \vartheta_{W}+12 \sin ^{4} \vartheta_{W} \simeq 0.911 \\
\sum_{\nu} C_{A}^{2}=\frac{3}{4}
\end{gathered}
$$

By modification of the method described in the introduction to weak processes, one obtains the following formula for the differential decay width :

$$
\begin{aligned}
d \Gamma= & \frac{G_{F}^{2}}{8 \omega_{1} \omega_{2}} \frac{d^{3} k_{1}}{(2 \pi)^{3}} \frac{d^{3} k_{2}}{(2 \pi)^{3}} \operatorname{Tr}\left(\left(\gamma k_{2}\right) \gamma_{\mu}\left(1-\gamma_{5}\right)\left(\gamma k_{1}\right) \gamma_{\nu}\left(1-\gamma_{5}\right)\right) \times \\
& \times \int_{-\infty}^{\infty} d \tau e^{-i \omega \tau}\left\langle i\left|\hat{Q}^{\mu \dagger}\left(\frac{\tau}{2}\right) \hat{Q}^{\nu}\left(-\frac{\tau}{2}\right)\right| i\right\rangle
\end{aligned}
$$

where $k_{1}=\left(\omega_{1}, \mathbf{k}_{1}\right)$ and $k_{2}=\left(\omega_{2}, \mathbf{k}_{2}\right)$ are the neutrino and antineutrino four-momenta, respectively, $\mid i>$ represents the initial quantum state of the electron and $\hat{Q}^{\mu}(t)$ denotes the following Heisenberg operator :

$$
\widehat{Q}^{\mu}(t)=\frac{\bar{u}_{f}(\widehat{p})}{(2 \widehat{H})^{1 / 2}} \gamma^{\mu}\left(C_{V}-C_{A} \gamma_{5}\right) \exp [-i \mathbf{k} \widehat{\mathbf{r}}(t)] \frac{u_{i}(\widehat{p})}{(2 \widehat{H})^{1 / 2}}
$$

with $q=(\omega, \mathbf{k})$ being the total four momentum of the neutrino pair $q=k_{1}+k_{2}$ and 


$$
u(\widehat{p})=\left(\begin{array}{c}
(\widehat{H}+m)^{1 / 2} w \\
(\widehat{H}+m)^{-1 / 2}(\sigma \widehat{\mathbf{p}}) w
\end{array}\right)
$$

the fourspinor operator representing the symbolic solution of the Dirac equation for a electron with a Hamiltonian $\widehat{H}$, which includes the effect of the external field. It can be obtained from the plane wave fourspinor solution by replacing $\mathbf{p}$ and $E$ to the operators $\widehat{\mathbf{p}}=\widehat{\mathbf{P}}-$ $e \mathbf{A}=-i \nabla-e \mathbf{A}, \widehat{H}=\left(\widehat{\mathbf{p}}^{2}+m^{2}\right)^{1 / 2}$. The spin state of the electron is determined by the spinor $w$. Finally, $\widehat{\mathbf{r}}(t)$ stands for the position operator of the electron.

By using the following identity

$$
\begin{aligned}
& \int \frac{d^{3} k_{1}}{2 \omega_{1}} \frac{d^{3} k_{2}}{2 \omega_{2}} \delta^{(4)}\left(q-k_{1}-k_{2}\right) \operatorname{Tr}\left(\left(\gamma k_{2}\right) \gamma_{\mu}\left(1-\gamma_{5}\right)\left(\gamma k_{1}\right) \gamma_{\nu}\left(1-\gamma_{5}\right)\right) \\
= & \frac{4 \pi}{3} \theta\left(q^{2}\right) \theta(\omega)\left(q_{\mu} q_{\nu}-g_{\mu \nu} q^{2}\right)
\end{aligned}
$$

one can reduce the number of integrations in Eq.(10)

$$
\begin{aligned}
d \Gamma= & \frac{4 \pi}{3} \frac{G_{F}^{2}}{2} \frac{d^{4} k}{(2 \pi)^{6}} \theta\left(q^{2}\right) \theta(\omega)\left(q_{\mu} q_{\nu}-g_{\mu \nu} q^{2}\right) \times \\
& \times \int_{-\infty}^{\infty} d \tau e^{-i \omega \tau}\left\langle i\left|\hat{Q}^{\mu \dagger}\left(\frac{\tau}{2}\right) \hat{Q}^{\nu}\left(-\frac{\tau}{2}\right)\right| i\right\rangle
\end{aligned}
$$

Since we assume the ultra-relativistic electron motion to be semi-classical, in the expression for $\widehat{Q}^{\mu}(t)$ only the non-commutativity of the electron operators with the neutrino field operator $\exp (-i \mathbf{k} \widehat{\mathbf{r}})$ needs to be taken into account. After this, we can replace the electron operators by their classical values. Following [9] we obtain:

$$
e^{-i \omega \tau} Q_{1}^{\mu \dagger} Q_{2}^{\nu} \approx \exp \left[i \frac{E}{E^{\prime}}\left(\mathbf{k r}_{2}-\mathbf{k r}_{1}-\omega \tau+\frac{\left(\omega^{2}-\mathbf{k}^{2}\right)}{2 E} \tau\right)\right] R_{2}^{* \mu} R_{1}^{\nu}
$$

with

$$
R^{\mu}(t)=\frac{u_{f}^{\dagger}\left(p^{\prime}\right)}{\left(2 E^{\prime}\right)^{1 / 2}} \gamma^{0} \gamma^{\mu}\left(C_{V}-C_{A} \gamma_{5}\right) \frac{u_{i}(p)}{(2 E)^{1 / 2}}
$$

here, $E^{\prime}=E-\omega$ and $\mathbf{p}^{\prime}(t)=\mathbf{p}(t)-\mathbf{k}$. We assume that the initial state of the electron has a momentum $\mathbf{p}(t)=E \mathbf{v}(t)$. We note that the last term in exponential (15) appears because $\omega^{2}-\mathbf{k}^{2} \geqslant 0$ for the radiated neutrino pair. Here and henceforward, suffixes 1 and 2 
denote the values of quantities at the times $t=-\frac{1}{2} \tau$ and $t=+\frac{1}{2} \tau$, respectively. Therefore, $\mathbf{r}_{1}$ and $\mathbf{r}_{2}$ are the electron coordinates at corresponding times.

An explicit calculation gives :

$$
\begin{gathered}
R^{0}=C_{V} \frac{w_{f}[2 E(E+m)-E(\mathbf{k} \cdot \mathbf{v})-i E((\mathbf{k} \times \mathbf{v}) \cdot \sigma)] w_{i}}{2 \sqrt{E E^{\prime}} \sqrt{\left(E^{\prime}+m\right)} \sqrt{(E+m)}}+ \\
+C_{A} \frac{w_{f}[2(E+m) E(\sigma \cdot \mathbf{v})-(E+m)(\sigma \cdot \mathbf{k})] w_{i}}{2 \sqrt{E E^{\prime}} \sqrt{\left(E^{\prime}+m\right)} \sqrt{(E+m)}} \\
\mathbf{R}=C_{V} \frac{w_{f}[(2 E+2 m-\omega) E \mathbf{v}-i \omega E(\mathbf{v} \times \sigma)+i(E+m)(\mathbf{k} \times \sigma)] w_{i}}{2 \sqrt{E E^{\prime}} \sqrt{\left(E^{\prime}+m\right)} \sqrt{(E+m)}} \\
C_{A} \frac{w_{f}\left[((2 m-\omega)(E+m)+E(\mathbf{k} \cdot \mathbf{v})) \sigma+2 E^{2}(\mathbf{v} \cdot \sigma) \mathbf{v}-E(\mathbf{k} \cdot \sigma) \mathbf{v}+i E(\mathbf{k} \times \mathbf{v})\right] w_{i}}{2 \sqrt{E E^{\prime}} \sqrt{\left(E^{\prime}+m\right)} \sqrt{(E+m)}}
\end{gathered}
$$

where the particle velocity $\mathbf{v}$ is a function of time $t$ when it moves in the magnetic field. As it follows from general considerations, the decay rate depends on the external magnetic field only through the combination 13

$$
\chi=\frac{p_{\perp}}{m} \frac{B}{B_{0}}
$$

Here $B_{0}=m^{2} / e$ is the critical Schwinger field and $p_{\perp}$ is the particle momentum component orthogonal to the external magnetic field. In the ultrarelativistic case under consideration one can write

$$
\chi=\frac{E}{m} \frac{B}{B_{0}} \sin \theta
$$

where $\theta$ is the angle between the magnetic field $\mathbf{B}$ and the particle momentum $\mathbf{p}$. The combination (19) is a Lorentz invariant. Therefore we can simplify our calculation by considering a particular reference frame where the electron moves along a circular trajectory orthogonal to the magnetic field. The general case can then be obtained by substituting $B \sin \theta$ instead of $B$ in the final result. Considering a circular electron trajectory, one has

$$
\begin{aligned}
& \mathbf{v}_{1}=\mathbf{v} \cos \left(\frac{\omega_{0} \tau}{2}\right)-(\mathbf{v} \times \mathbf{h}) \sin \left(\frac{\omega_{0} \tau}{2}\right) \\
& \mathbf{v}_{2}=\mathbf{v} \cos \left(\frac{\omega_{0} \tau}{2}\right)+(\mathbf{v} \times \mathbf{h}) \sin \left(\frac{\omega_{0} \tau}{2}\right)
\end{aligned}
$$


with $\mathbf{h}$ being the unit vector along the magnetic field. As it follows from kinematics, the emission in a given direction $\mathbf{k}$ is produced on a small part of the path in which $\mathbf{v}$ turns, in the case of $\chi \lesssim 1$, an angle $\sim m / E$ or, in the case of $\chi \gg 1$, an angle $\sim \chi^{1 / 3} m / E$. This length is traversed on a time interval $\tau \ll \omega_{0}^{-1}$. This short time interval gives the main contribution to the integral over $d \tau$. Expansion in powers of $\omega_{0} \tau$ yields:

$$
\mathbf{k}\left(\mathbf{r}_{2}-\mathbf{r}_{1}\right) \simeq \mathbf{k v} \tau-\frac{\mathbf{k v}}{24} \tau^{3} \omega_{0}^{2}
$$

where $\mathbf{v}$ is the electron velocity at $t=0$. We can replace $\mathbf{k v}$ by $\omega$ in the last term in (23) because $\omega-\mathbf{k v} \ll \omega$. Thus we obtain

$$
\exp \left[i \frac{E}{E^{\prime}}\left(\mathbf{k r}_{2}-\mathbf{k} \mathbf{r}_{1}-\omega \tau\right)\right] \approx \exp \left[i \frac{E}{E^{\prime}}\left(\mathbf{k} \mathbf{v} \tau-\omega \tau+\frac{\left(\omega^{2}-\mathbf{k}^{2}\right)}{2 E} \tau-\frac{\omega}{24} \tau^{3} \omega_{0}^{2}\right)\right]
$$

We introduce now the following notation

$$
\begin{aligned}
M^{\dagger} M & \equiv\left\langle i\left|\hat{Q}^{\mu \dagger}\left(\frac{\tau}{2}\right) \hat{Q}^{\nu}\left(-\frac{\tau}{2}\right)\right| i\right\rangle\left(q_{\mu} q_{\nu}-g_{\mu \nu} q^{2}\right) \\
& =\frac{4 \pi}{3}\left(q_{\mu} q_{\nu}-g_{\mu \nu} q^{2}\right) \frac{1}{2} \sum_{i} \sum_{f} R_{2}^{* \mu} R_{1}^{\nu}
\end{aligned}
$$

Summation over the final electron polarization and averaging over the initial electron (which we consider non-polarized) can be performed with the help of polarization density matrices for the initial and final electron. Keeping terms with an accuracy of $m^{4} / E^{4}$ we obtain:

$$
\begin{aligned}
M^{\dagger} M= & \frac{4 \pi}{3}\left(C_{V}^{2}+C_{A}^{2}\right) \frac{E^{2}}{\left(E^{\prime}\right)^{2}}\left[(\omega-\mathbf{k v})^{2}-\right. \\
& \left.-\left(\omega^{2}-k^{2}\right)\left(\frac{E^{2}+\left(E^{\prime}\right)^{2}}{2 E^{2}} \frac{m^{2}}{E^{2}}+\frac{E^{\prime}}{E^{2}}(\omega-\mathbf{k v})+\frac{\left(\omega^{2}-k^{2}\right)}{4 E^{2}}-\frac{m^{2} \omega^{2}}{2 E^{4}}\right)\right]+ \\
& +\frac{4 \pi}{3}\left(C_{V}^{2}+C_{A}^{2}\right) \frac{E^{2}}{\left(E^{\prime}\right)^{2}}\left\{\left[\mathbf{k v}(\omega-\mathbf{k v})-(\mathbf{k}(\mathbf{v} \times \mathbf{h}))^{2}-\right.\right. \\
& \left.\left.-\left(\omega^{2}-k^{2}\right)\left(\left(\frac{E^{2}+\left(E^{\prime}\right)^{2}}{E^{2}}\right)+\frac{E^{\prime}}{E^{2}} \frac{1}{2} \mathbf{k v}\right)\right] \frac{\tau^{2} \omega_{0}^{2}}{4}+\omega^{2} \frac{\tau^{4} \omega_{0}^{4}}{64}\right\}+ \\
& +\frac{4 \pi}{3} C_{A}^{2} \frac{m^{2}}{E^{\prime 2}}\left[3 \frac{E^{\prime}}{E}\left(\omega^{2}-k^{2}\right)-\omega^{2} \frac{\tau^{2} \omega_{0}^{2}}{2}\right]+ \\
& -\frac{4 i \pi}{3} C_{V} C_{A} \frac{1}{\left(E^{\prime}\right)^{2}}\left(E^{\prime}+m\right)\left(\omega^{2}-k^{2}\right)(\mathbf{k h}) \tau \omega_{0}
\end{aligned}
$$

Substitution of Eqs.(24) and (26) into Eq.(14) yields 


$$
\begin{aligned}
d \Gamma= & \frac{G_{F}^{2}}{2} \frac{d \omega}{(2 \pi)^{6}} \int_{0}^{\omega} d k k^{2} d \Omega \\
& \times \int_{-\infty}^{\infty} d \tau \exp \left[-i \frac{E}{E^{\prime}}\left(\left(\omega-\mathbf{k v}+\frac{\left(\omega^{2}-\mathbf{k}^{2}\right)}{2 E}\right) \tau+\frac{\omega}{24} \tau^{3} \omega_{0}^{2}\right)\right] M^{\dagger} M
\end{aligned}
$$

The last term in Eq.(26) contributes only to the azimuthal distribution of radiated neutrino pairs, and will vanish after integration over all directions of $\mathbf{k}$ with respect to the initial electron velocity : $d \Omega=\sin (\vartheta) d \vartheta d \varphi$. Integration over $d \varphi$ is trivial. In order to perform the next integrations let us use instead of $\omega$ the new variable

$$
s \equiv \frac{1}{\gamma^{2}}\left(\frac{E \omega}{E^{\prime} \omega_{0}}\right)^{2 / 3}
$$

where $\gamma=m / E$ is the electron Lorentz factor, so that

$$
\frac{\omega}{E}=\frac{\chi s^{3 / 2}}{\left(1+\chi s^{3 / 2}\right)}
$$

and introduce the following changes of variable :

$$
\begin{gathered}
z \equiv \frac{2}{\omega} \gamma^{2} s\left(\omega-k v \cos \vartheta-\frac{\omega^{2}-k^{2}}{2 E}\right) \\
\frac{u^{3}}{3} \equiv \frac{E}{E^{\prime}} \frac{\omega \omega_{0}^{2}}{24} \tau^{3} \\
a \equiv \frac{2}{\omega}\left(\omega-k v-\frac{\omega^{2}-k^{2}}{2 E}\right) \gamma^{2} s
\end{gathered}
$$

Taking also into account the following identity for the Airy function and its $n$-th derivatives

$$
\int_{-\infty}^{\infty} d u \cdot u^{n} \exp \left[-i\left(x u+\frac{u^{3}}{3}\right)\right]=2 \pi(i)^{n} \stackrel{(n)}{\mathrm{Ai}}(x)
$$

one obtains, with leading accuracy $1 / \gamma^{6}$

$$
\begin{aligned}
& \frac{d \Gamma}{d s}=\frac{G_{F}^{2} m^{4}}{16(2 \pi)^{3}} \frac{m}{\gamma} \frac{\chi^{5} s^{3+1 / 2}}{\left(1+\chi s^{3 / 2}\right)^{4}} \int_{s}^{\infty} d a \int_{a}^{\infty} d z \times \\
& \left\{\left(C_{V}^{2}+C_{A}^{2}\right)\left[2 \frac{\chi^{2} s^{3}}{\left(1+\chi s^{3 / 2}\right)}(a-s)(s+z-a)+z^{2}-4 s(a-s)\right] \mathrm{Ai}(z)\right. \\
& +\left(C_{V}^{2}+C_{A}^{2}\right)\left\{\left[\left(6+2 \chi s^{3 / 2}-2 \frac{\chi s^{3 / 2}}{\left(1+\chi s^{3 / 2}\right)}\right)(a-s) \stackrel{(2)}{\mathrm{Ai}(z)-2 s \mathrm{Ai}(z)]}\right.\right. \\
& \left.+\stackrel{(4)}{\mathrm{Ai}}(z)\}+4 C_{A}^{2}[3 s(a-s) \mathrm{Ai}(z)+2 s \stackrel{(2)}{\mathrm{Ai}}(z)]\right\}
\end{aligned}
$$


We have replaced the upper limit of integration over $d z$ by infinity, because the actual limit is given by

$$
\frac{2}{\omega}\left(\omega+k v-\frac{\omega^{2}-k^{2}}{2 E}\right) \gamma^{2} s \simeq 2\left(2-\frac{\omega^{2}-k^{2}}{2 E \omega}\right) \gamma^{2} s \simeq 4 \gamma^{2} s \gg 1
$$

(see Eq.(28)). In this upper limit, the Airy function tends to zero exponentially. Performing integration by parts we get

$$
\begin{aligned}
\frac{d \Gamma}{d s}= & \frac{G_{F}^{2} m^{4}}{16(2 \pi)^{3}} \frac{m}{\gamma} \frac{\chi^{5} s^{3+1 / 2}}{\left(1+\chi s^{3 / 2}\right)^{4}} \times \\
& \left\{( C _ { V } ^ { 2 } + C _ { A } ^ { 2 } ) \left[\frac{\chi^{2} s^{3}}{\left(1+\chi s^{3 / 2}\right)} \int_{s}^{\infty}\left[2+\frac{1}{3}(2 s+a)(a-s)^{2}\right] \mathrm{Ai}(a) d a\right.\right. \\
& \left.+\int_{s}^{\infty}\left[6+(a-s)\left(s^{2}+(s-a)^{2}\right)\right] \mathrm{Ai}(a) d a-s \operatorname{Ai}(s)\right] \\
& \left.+C_{A}^{2} 8 s\left[\frac{3}{4}\left(\int_{s}^{\infty}(s-a)^{2} \operatorname{Ai}(a) d a\right)+\operatorname{Ai}(s)\right]\right\}
\end{aligned}
$$

Since $s$, defined by Eq.(28), is a function of $\omega$, this formula represents the energy spectrum of radiated neutrino pairs.

In Fig. 2 we have plotted the neutrino pair energy spectrum, as a function of the variable $s$, obtained from Eq.(36) (normalized so that the integral over $s$ is equal to unity in all cases), for three values of the characteristic parameter $\chi$. The energy distribution has a maximum for $s \sim 1$ if $\chi \ll 1$. Then, in this limit, radiated neutrinos and antineutrinos have energies $\omega \sim \omega_{0} \gamma^{3}$, while for $\chi \gg 1$ the energy distribution peaks at small $s \sim \chi^{-2 / 3}$. The energy of the emitted neutrino pair, in this case, goes up to $\omega \sim E$, while the energy of the final particle is as small as $m B_{0} / B$. By this reason, in the ultrarelativistic limit under consideration, we have to require $B \ll B_{0}$. To obtain the total decay width, Eq.(36) must be integrated with respect to $s$ from 0 to $\gamma / \chi$, as follows from Eq.(29) when $\omega$ varies from 0 to $E-m \simeq E$.

$$
\begin{aligned}
\Gamma= & \frac{G_{F}^{2} m^{4}}{16(2 \pi)^{3}} \frac{m}{\gamma} \int_{0}^{\gamma / \chi} d s \frac{\chi^{5} s^{3+1 / 2}}{\left(1+\chi s^{3 / 2}\right)^{4}} \times \\
& \left\{( C _ { V } ^ { 2 } + C _ { A } ^ { 2 } ) \left[\frac{\chi^{2} s^{3}}{\left(1+\chi s^{3 / 2}\right)} \int_{s}^{\infty}\left[2+\frac{1}{3}(2 s+a)(a-s)^{2}\right] \operatorname{Ai}(a) d a\right.\right. \\
& \left.+\int_{s}^{\infty}\left[6+(a-s)\left(s^{2}+(s-a)^{2}\right)\right] \operatorname{Ai}(a) d a-s \operatorname{Ai}(s)\right]
\end{aligned}
$$




$$
\left.+C_{A}^{2} 8 s\left[\frac{3}{4}\left(\int_{s}^{\infty}(s-a)^{2} \mathrm{Ai}(a) d a\right)+\mathrm{Ai}(s)\right]\right\}
$$

Since we assume $\gamma / \chi=B_{0} / B \gg 1$, integration can be extended up to infinity in the latter equation.

The total width can be calculated analytically in two limiting cases of small and large value of $\chi$, by taking into account the above discussion. When $\chi \ll 1$ we obtain :

$$
\Gamma(\chi \ll 1)=\frac{49}{27} \sqrt{3} \frac{\left(C_{V}^{2}+C_{A}^{2}\right) G_{F}^{2}}{16(2 \pi)^{3}} \frac{m^{6}}{E} \chi^{5}+\frac{7}{4} \sqrt{3} \frac{C_{A}^{2} G_{F}^{2}}{2(2 \pi)^{3}} \frac{m^{6}}{E} \chi^{5}
$$

This result coincides with that given by Baier and Katkov [10] for $\nu \bar{\nu}$ synchrotron radiation of an electron, if we take $C_{V}=C_{A}=1$. In the opposite case of extremely large $\ln \chi \gg 1$, we get the leading term

$$
\Gamma(\chi \gg 1)=\frac{\left(C_{V}^{2}+C_{A}^{2}\right) G_{F}^{2}}{27(2 \pi)^{3}} \frac{m^{6}}{E} \chi^{2} \ln \chi
$$

which also coincides with that obtained by Baier and Katkov [10] (see also [11] and [12])

\section{SINGLE-PARTICLE APPROXIMATION}

Consider now the neutrino pair emissivity due to synchrotron radiation from a magnetized ultrarelativistic degenerate electron gas. Here some remarks are in order. When calculating the rate of $\nu \bar{\nu}$ synchrotron emission by an ultrarelativistic electron in vacuum we required $B \ll B_{0}$ in order to the final electron still be ultrarelativistic. Now, when we consider a degenerate electron gas, one can omit this limitation because the radiated pair energy is of the order of the medium temperature and, consequently, it is much smaller than the energy of the initial electron, which comes from the vicinity of the Fermi surface. To use the above developed method in this case, one requires only two conditions: the electron must be ultrarelativistic, i.e. $E_{F} \gg m$, and its motion must be semi-classical, i.e. $\omega_{0} \ll E_{F}$. The latter condition is equivalent to $B \ll B_{0} E_{F}^{2} / m^{2}$.

As we will show, in this scenario calculations are essentially simpler than those for vacuum, because in the matrix elements one can neglect all terms which are of the order of, or 
smaller than, $T / E_{F}$. Then the contribution of the contact weak interaction can be simply obtained from Eq.(36) if one takes into account that, under the conditions we considered, $\chi s^{3 / 2} \sim T / E_{F} \ll 1$. Neglecting these small terms one obtains

$$
\begin{aligned}
\frac{d \Gamma}{d s}= & \frac{G_{F}^{2} m^{4}}{16(2 \pi)^{3}} \frac{m}{\gamma} \chi^{5} s^{7 / 2}\left\{( C _ { V } ^ { 2 } + C _ { A } ^ { 2 } ) \left[\int_{s}^{\infty}\left[6+(a-s)\left(s^{2}+(s-a)^{2}\right)\right] \operatorname{Ai}(a) d a\right.\right. \\
& \left.-s \operatorname{Ai}(s)]+C_{A}^{2} 8 s\left[\int_{s}^{\infty}(s-a)^{2} \operatorname{Ai}(a) d a+\operatorname{Ai}(s)\right]\right\}
\end{aligned}
$$

In the actual magnetic field of a neutron star one has, moreover,

$$
B \gg \frac{m T}{E_{F}^{2}} B_{0}
$$

Therefore

$$
s \equiv \frac{m^{2}}{E_{F}^{2}}\left(\frac{\omega}{\omega_{0}}\right)^{2 / 3} \sim\left(\frac{m T}{E_{F}^{2}} \frac{B_{0}}{B}\right)^{2 / 3} \ll 1
$$

and one can substitute by zero the lower limit of integration in (40) and neglect $s$ inside the integrals. Then one can perform this integral analytically. Keeping only leading terms

and making the substitution (20), we obtain the rate of neutrino pair emission due to the contact weak interaction

$$
\frac{d \Gamma_{w}}{d \omega}=\left(C_{V}^{2}+C_{A}^{2}\right) \frac{G_{F}^{2} m^{4}}{9(2 \pi)^{3}} n(E)[1-n(E-\omega)] \frac{B^{2}}{B_{0}^{2}} \frac{\omega^{2}}{E_{F}^{2}} \sin ^{2} \theta
$$

Here we introduced the electron statistical factors $n(1-n)$ with

$$
n(E) \simeq \frac{1}{e^{\left(E-E_{F}\right) / T}+1}
$$

taking into account the Pauli principle.

\section{IN-MEDIUM EFFECTIVE WEAK INTERACTION}

As it follows from the second Feynman diagram, the effective interaction of neutrinos with the photon field $A_{\mu}$ arises from the medium polarization coupling to the weak neutrino current $\bar{\nu} \gamma_{\mu}\left(1-\gamma_{5}\right) \nu$. Since one can neglect the magnetic field contribution to the medium polarization, the effective vertex of this interaction reads (see also [14], [15]) 


$$
\frac{G_{F}}{\sqrt{2}} \Gamma^{\mu \nu}=\frac{G_{F}}{\sqrt{2}} \frac{1}{\sqrt{e^{2}}}\left(C_{V} \Pi_{l}(q) e^{\mu} e^{\nu}+g^{\mu i}\left[C_{V} \Pi_{t}(q)\left(\delta^{i j}-n^{i} n^{j}\right)+i C_{A} \Pi_{A}(q) \epsilon^{i j m} n^{m}\right] g^{j \nu}\right)
$$

with the notations $\mathbf{n}=\mathbf{k} / k, k=|\mathbf{k}|$ and

$$
e^{\mu}=\left(1, \frac{\omega}{k} \mathbf{n}\right)
$$

$\Pi_{l}(q)$ and $\Pi_{t}(q)$ are the longitudinal and transversal electromagnetic polarization functions, respectively, and $\Pi_{A}(q)$ is the axial polarization function . These functions, calculated in the one-loop approximation for an ultrarelativistic degenerate electron gas are [15]:

$$
\begin{gathered}
\Pi_{l}(q)=3 \omega_{p}^{2} \frac{\omega^{2}}{k^{2}}\left(\frac{\omega}{2 k} \ln \frac{\omega+k}{\omega-k}-1\right) \\
\Pi_{t}(q)=\frac{3}{2} \omega_{p}^{2} \frac{\omega^{2}}{k^{2}}\left(1-\frac{\omega^{2}-k^{2}}{\omega^{2}} \frac{\omega}{2 k} \ln \frac{\omega+k}{\omega-k}\right) \\
\Pi_{A}(q)=\frac{3}{2} \frac{\omega_{p}^{2}}{E_{F}} \frac{\omega^{2}-k^{2}}{k}\left(\frac{\omega}{2 k} \ln \frac{\omega+k}{\omega-k}-1\right)
\end{gathered}
$$

where $\omega_{p}$ is the plasma frequency. One can see that, when $q^{2} / \omega_{p}^{2} \ll 1$, the longitudinal and transversal polarization functions behave as :

$$
\begin{gathered}
\Pi_{l}\left(q^{2} \rightarrow 0\right)=\frac{3}{2} \omega_{p}^{2} \ln \frac{4 \omega^{2}}{q^{2}} \\
\Pi_{t}\left(q^{2} \rightarrow 0\right)=\frac{3}{2} \omega_{p}^{2}
\end{gathered}
$$

while the axial polarization function goes to zero

$$
\Pi_{A}\left(q^{2} \rightarrow 0\right)=\frac{3}{4} \frac{\omega_{p}^{2}}{E_{F}} \frac{q^{2}}{\omega} \ln \frac{4 \omega^{2}}{q^{2}} \rightarrow 0
$$

and, by this reason, it can be neglected. So, instead of (18) one should write

$$
\mathcal{R}^{\mu}(t)=R^{\mu}(t)+r^{\mu}(t)
$$

where $R^{\mu}(t)$ is given by Eq. (16), while 


$$
r^{\mu}(t)=\frac{u_{f}^{\dagger}\left(p^{\prime}\right)}{\left(2 E^{\prime}\right)^{1 / 2}} \gamma^{0} \gamma^{\lambda} \frac{u_{i}(p)}{(2 E)^{1 / 2}} D_{\lambda \rho}(q) \Gamma^{\rho \mu}(q)
$$

is the matrix element corresponding to the second diagram. Here $D_{\lambda \rho}(q)$ is the effective propagator for the electromagnetic field in the medium, which in the $A_{0}=0$ gauge reads

$$
D_{\lambda \rho}(q)=g_{i \lambda}\left[-\frac{1}{\omega^{2}-\Pi_{l}} n^{i} n^{j}-\frac{1}{q^{2}-\Pi_{t}}\left(\delta^{i j}-n^{i} n^{j}\right)\right] g_{j \rho}
$$

As it follows from Eq. (27) the rate of neutrino pair emission is

$$
\begin{aligned}
d \Gamma= & \frac{G_{F}^{2}}{2} \frac{d \omega}{(2 \pi)^{6}} n(E)[1-n(E-\omega)] \int_{0}^{\omega} d k k^{2} d \Omega \\
& \times \int_{-\infty}^{\infty} d \tau \exp \left[-i\left((\omega-\mathbf{k v}) \tau+\frac{\omega}{24} \tau^{3} \omega_{0}^{2}\right)\right](M+\mu)^{\dagger}(M+\mu)
\end{aligned}
$$

We have introduced the statistical factors $n(E)[1-n(E-\omega)]$, as in previous section, and neglected the third term in the exponential (see Eq.(24)) because $\omega \sim T \ll E$. As it follows from (55), one has to evaluate the contribution of three terms: the first one is the contribution from contact weak interaction, defined by

$$
M^{\dagger} M=\frac{4 \pi}{3} \frac{1}{2} \sum_{i} \sum_{f} \operatorname{Tr}\left(R_{2}^{* \mu} R_{1}^{\nu}\right)\left(q_{\mu} q_{\nu}-g_{\mu \nu} q^{2}\right)
$$

The second one is the contribution of the effective interaction via the intermediate virtual photon

$$
\mu^{\dagger} \mu=\frac{4 \pi}{3} \frac{1}{2} \sum_{i} \sum_{f} \operatorname{Tr}\left(r_{2}^{* \mu} r_{1}^{\nu}\right)\left(q_{\mu} q_{\nu}-g_{\mu \nu} q^{2}\right)
$$

and the third one is the interferential term, given by

$$
M^{\dagger} \mu+\mu^{\dagger} M=\frac{4 \pi}{3} \frac{1}{2} \sum_{i} \sum_{f} \operatorname{Tr}\left(R_{2}^{* \mu} r_{1}^{\nu}+r_{2}^{* \mu} R_{1}^{\nu}\right)\left(q_{\mu} q_{\nu}-g_{\mu \nu} q^{2}\right)
$$

The first contribution has been already evaluated, and one has now to calculate the rate of neutrino pair emission due to weak interactions, via the intermediate virtual photon, and the interferential term. 


\section{COLLECTIVE EFFECTS}

In accordance to (53) one has

$$
r^{\mu}=-C_{V} \xi^{i}\left[\frac{\omega}{k} \frac{\Pi_{l}}{\omega^{2}-\Pi_{l}} n^{i} e^{\mu}-\frac{\Pi_{t}}{q^{2}-\Pi_{t}}\left(\delta^{i j}-n^{i} n^{j}\right) g^{j \mu}\right]
$$

We introduced here a short notation for the following matrix element

$$
\xi=\frac{u_{f}^{*}\left(p^{\prime}\right)}{\left(2 E^{\prime}\right)^{1 / 2}} \alpha \frac{u_{i}(p)}{(2 E)^{1 / 2}}
$$

Taking into account $r^{\mu} q_{\mu}=0$, contraction in (57) yields

$$
\begin{aligned}
& \frac{4 \pi}{3} r_{2}^{* \mu} r_{1}^{\nu}\left(q_{\mu} q_{\nu}-g_{\mu \nu} q^{2}\right) \\
= & \frac{4 \pi}{3} C_{V}^{2} q^{2}\left[\frac{\omega^{2} q^{2}}{k^{4}}\left|\frac{\Pi_{l}}{\omega^{2}-\Pi_{l}}\right|^{2}\left(\xi_{2}^{*} \mathbf{n}\right)\left(\xi_{1} \mathbf{n}\right)+\left|\frac{\Pi_{t r}}{q^{2}-\Pi_{t r}}\right|^{2}\left(\left(\xi_{2}^{*} \xi_{1}\right)-\left(\xi_{2}^{*} \mathbf{n}\right)\left(\xi_{1} \mathbf{n}\right)\right)\right]
\end{aligned}
$$

Since in the case of a degenerate electron gas, the energy of the radiated neutrino pair $\omega \sim T$ is much smaller than the initial electron energy $E \simeq E_{F}$, one can neglect in the matrix elements (60) all terms which are of the order or smaller than $m T / E_{F}^{2}$. Then one obtains

$$
\xi=\left(w_{f}^{*} w_{i}\right) \mathbf{v}(t)
$$

Summation in (61) over the final electron polarization and averaging over the initial electron yields

$$
\mu^{\dagger} \mu=\frac{4 \pi}{3} C_{V}^{2} q^{2}\left[\frac{\omega^{2} q^{2}}{k^{4}}\left|\frac{\Pi_{l}}{\omega^{2}-\Pi_{l}}\right|^{2}\left(\mathbf{v}_{2} \mathbf{n}\right)\left(\mathbf{v}_{1} \mathbf{n}\right)+\left|\frac{\Pi_{t r}}{\omega^{2}-\Pi_{t r}}\right|^{2}\left[\mathbf{v}_{2} \mathbf{v}_{1}-\left(\mathbf{v}_{2} \mathbf{n}\right)\left(\mathbf{v}_{1} \mathbf{n}\right)\right]\right]
$$

Expanding $\mathbf{v}_{2}$ and $\mathbf{v}_{1}$ in the small parameter $\omega_{0}^{2} \tau^{2}$ we obtain, according to Eqs. (21,22)

$$
\mu^{\dagger} \mu=\frac{4 \pi}{3} C_{V}^{2} q^{2}\left[\frac{q^{2}}{\omega^{2}}\left|\frac{\Pi_{l}}{\omega^{2}-\Pi_{l}}\right|^{2}(\mathbf{n v})^{2}+\left|\frac{\Pi_{t r}}{q^{2}-\Pi_{t r}}\right|^{2}\left(\mathbf{v}^{2}-(\mathbf{n v})^{2}-\mathbf{v}^{2} \frac{\omega_{0}^{2} \tau^{2}}{4}\right)\right]
$$

In the fist term in the square brackets we keep only $(\mathbf{n v})^{2} \sim 1$ because this term is multiplied by $q^{2} / \omega^{2} \sim 1 / \gamma^{2}$.

We introduce a special notation $\Gamma_{e m}$ for the second diagram contribution to the rate of reaction in order to distinguish it from $\Gamma_{w}$. Performing integrations in 


$$
\begin{aligned}
\frac{d \Gamma_{e m}}{d \omega}= & \frac{G_{F}^{2}}{2} \frac{1}{(2 \pi)^{6}} n(E)[1-n(E-\omega)] \times \\
& \times \int_{0}^{\omega} d k k^{2} \int d \Omega \int_{-\infty}^{\infty} d \tau \exp \left[-i\left((\omega-k v x) \tau+\frac{\omega}{24} \tau^{3} \omega_{0}^{2}\right)\right] \mu^{\dagger} \mu
\end{aligned}
$$

as it was made in the previous section, we arrive to the following expression

$$
\begin{aligned}
\frac{d \Gamma_{e m}}{d \omega}= & \frac{G_{F}^{2} C_{V}^{2}}{2} \frac{1}{(2 \pi)^{4}} n(E)[1-n(E-\omega)] \int_{0}^{\omega} d k k \int_{a}^{a_{1}} d z \\
& \frac{4 \pi}{3} q^{2}\left\{\frac{q^{2} k^{2}}{\omega^{4}}\left|\frac{\Pi_{l}}{\omega^{2}-\Pi_{l}}\right|^{2} \frac{\omega^{2}}{v^{2} k^{2}}(1-\beta z)^{2} \mathrm{Ai}(z)\right. \\
& \left.+\left|\frac{\Pi_{t r}}{q^{2}-\Pi_{t r}}\right|^{2}\left(\left(1-\frac{\omega^{2}}{v^{2} k^{2}}(1-\beta z)^{2}\right) \operatorname{Ai}(z)+2 \beta \operatorname{Ai}(z)\right)\right\}
\end{aligned}
$$

with the notation

$$
\beta \equiv \frac{1}{2}\left(\frac{\omega_{0}}{\omega}\right)^{2 / 3}
$$

and where the limits of integration over $d z$ are

$$
\begin{aligned}
& a=\frac{2}{\omega}\left(\frac{\omega}{\omega_{0}}\right)^{2 / 3}(\omega-k v) \\
& a_{1}=\frac{2}{\omega}\left(\frac{\omega}{\omega_{0}}\right)^{2 / 3}(\omega+k v)
\end{aligned}
$$

Since we assume

$$
\left(\frac{\omega}{\omega_{0}}\right)^{2 / 3}=\left(\frac{\omega E_{F}}{e B}\right)^{2 / 3} \sim\left(\frac{T E_{F}}{e B}\right)^{2 / 3} \gg 1
$$

one can change the upper limit of integration over $d z$ to infinity because the Airy function exponentially tends to zero when $z \gg 1$. By the same reason, the integrand is not small only if the lower limit is of the order or less than unity. This means that only those values of $k$ which are very close to $\omega$ contribute dominantly to the integral

$$
\frac{(\omega-k)}{\omega} \sim\left(\frac{\omega_{0}}{\omega}\right)^{2 / 3} \ll 1
$$

The latter inequality also means that 


$$
q^{2} \simeq 2 \omega(\omega-k) \sim \omega^{2}\left(\frac{\omega_{0}}{\omega}\right)^{2 / 3} \ll \omega^{2}
$$

Therefore one can expand the medium polarization function, by keeping only leading terms for $q^{2} \rightarrow 0$ as was made in (49, 50), and rewrite (66) as follows

$$
\begin{aligned}
\frac{d \Gamma_{e m}}{d \omega}= & \frac{G_{F}^{2} C_{V}^{2}}{6(2 \pi)^{3}} n(E)[1-n(E-\omega)] \omega_{0}^{2} \omega^{2} \int_{s}^{\infty} d a \int_{a}^{\infty} d z \\
& {\left[a^{2}\left|F_{l}(\omega, a)\right|^{2} \operatorname{Ai}(z)+\left|F_{t r}(\omega, a)\right|^{2}((z-a) a \operatorname{Ai}(z)+a \stackrel{(2)}{\mathrm{Ai}}(z))\right] }
\end{aligned}
$$

where

$$
\omega^{2}-k^{2} \simeq \omega^{2}\left(\frac{\omega_{0}}{\omega}\right)^{2 / 3} a
$$

was used. With good accuracy one has

$$
F_{t r}(\omega, a) \equiv-\lim _{q^{2} \rightarrow 0} \frac{\Pi_{t r}}{q^{2}-\Pi_{t r}} \simeq 1
$$

while

$$
F_{l}(\omega, a) \equiv-\lim _{q^{2} \rightarrow 0} \frac{\Pi_{l}}{\omega^{2}-\Pi_{l}}=-\frac{\omega_{p}^{2}\left(\ln \left(\frac{\omega}{\omega_{0}}\right)+\frac{3}{2} \ln \frac{4}{a}\right)}{\omega^{2}-\omega_{p}^{2}\left(\ln \left(\frac{\omega}{\omega_{0}}\right)+\frac{3}{2} \ln \frac{4}{a}\right)}
$$

We focus on the condition

$$
\frac{T^{2}}{\omega_{p}^{2}} \ll \ln \left(\frac{T}{\omega_{0}}\right)
$$

which is typically valid for a long epoch of neutron star cooling. Then one can also substitute $F_{l}(\omega, a) \simeq 1$.Integration by parts can then be performed. This yields

$$
\frac{d \Gamma_{e m}}{d \omega}=C_{V}^{2} \frac{G_{F}^{2} m^{4}}{9(2 \pi)^{3}}\left(\frac{B}{B_{0}} \sin \theta\right)^{2} n(E)[1-n(E-\omega)] \frac{\omega^{2}}{E_{F}^{2}}
$$

To evaluate the interferential term we neglect in (17) and (18) all terms which are proportional to $\omega$ and $k$, because $\omega \sim T \ll E_{F}$, and we omit all terms proportional to $\sigma$ matrices, because $\operatorname{Tr} \sigma=0$. Then instead of (17) and (18) we get

$$
R^{\mu}=C_{V}(1, \mathbf{v}(t))\left(w_{f}^{*} w_{i}\right)
$$


Taking also into account (59) and (62), we obtain

$$
\begin{aligned}
\mu^{\dagger} M+M^{\dagger} \mu= & \frac{4 \pi}{3} \frac{1}{2} \sum_{i} \sum_{f}\left\langle i\left|\left(\mathbf{R}_{2}^{* \mu} \mathbf{r}_{1}^{\nu}+\left(\mathbf{r}_{2}^{\mu} \mathbf{R}_{1}^{* \nu}\right)\right)\right| i\right\rangle\left(q_{\mu} q_{\nu}-g_{\mu \nu} q^{2}\right)= \\
& \frac{4 \pi}{3} C_{V} q^{2}\left[\frac{\omega}{k} \frac{\Pi_{l}}{\omega^{2}-\Pi_{l}}\left[\left(\mathbf{v}_{2} \mathbf{n}-1\right)\left(\mathbf{v}_{1} \mathbf{n}\right)+\left(\mathbf{v}_{1} \mathbf{n}-1\right)\left(\mathbf{v}_{2} \mathbf{n}\right)\right]\right. \\
& \left.\left.+\frac{\Pi_{t}}{q^{2}-\Pi_{t}} 2\left(\mathbf{v}_{2} \mathbf{v}_{1}-\left(\mathbf{v}_{1} \mathbf{n}\right)\left(\mathbf{v}_{2} \mathbf{n}\right)\right)\right]\right]
\end{aligned}
$$

Expansion of $\mathbf{v}_{2}$ and $\mathbf{v}_{1}$ in the small parameter $\omega_{0}^{2} \tau^{2}$ and performing integrations analogously to those in (65) we arrive to the following expression for the interferential term:

$$
\frac{d \Gamma_{i n t}}{d \omega}=\frac{5}{6} \frac{C_{V}^{2} G_{F}^{2}}{9(2 \pi)^{3}}\left(\frac{B}{B_{0}} \sin \theta\right)^{2} n(E)[1-n(E-\omega)] \frac{\omega^{2}}{E_{F}^{2}}
$$

Collecting (43), (78) and (82) we obtain the following expression for the deferential rate of $\nu \bar{\nu}$ synchrotron radiation from the degenerate ultrarelativistic electron gas of a neutron star

$$
\frac{d \Gamma}{d \omega}=\left(\frac{17}{6} C_{V}^{2}+C_{A}^{2}\right) \frac{G_{F}^{2} m^{4}}{9(2 \pi)^{3}} \frac{B^{2}}{B_{0}^{2}} n(E)[1-n(E-\omega)] \frac{\omega^{2}}{E_{F}^{2}} \sin ^{2} \theta
$$

Comparison of this formula with (43) shows that, due to collective effects under consideration, the contribution of vector weak currents to the rate of neutrino pair emission is almost three times more than that calculated in the single particle approximation.

\section{NEUTRINO PAIR EMISSIVITY}

With the help of Eq.(83) one can calculate neutrino pair emissivity due to the synchrotron process in the regime we are considering

$$
Q_{\nu}=2 \int \frac{d^{3} p}{(2 \pi)^{3}} d \omega \omega\left(\frac{17}{6} \sum_{\nu} C_{V}^{2}+\sum_{\nu} C_{A}^{2}\right) \frac{G_{F}^{2} m^{4}}{9(2 \pi)^{3}} \frac{B^{2}}{B_{0}^{2}} n(E)[1-n(E-\omega)] \frac{\omega^{2}}{E_{F}^{2}} \sin ^{2} \theta
$$

Here summation is performed over three neutrino species, see (8, 9) and the extra factor 2 takes into account summation over the initial electron polarization. Because of the blocking

factor only electrons from a vicinity of the Fermi surface contribute to the integral, so one can use the following approximation 


$$
d^{3} p=p^{2} d p \sin \theta d \theta d \varphi \simeq p_{F} E_{F} d E \sin \theta d \theta d \varphi
$$

Then integrals become trivial and we obtain

$$
Q_{\nu}=\frac{2 \zeta(5)}{9 \pi^{5}}\left(\frac{17}{6} \sum_{\nu} C_{V}^{2}+\sum_{\nu} C_{A}^{2}\right) G_{F}^{2} m^{4} T^{5} \frac{B^{2}}{B_{0}^{2}}
$$

A comparison with the result obtained in [5] shows that, due to collective effects, the contribution of vector weak currents to the neutrino pair emissivity is $17 / 6$ times larger than that calculated in the single-particle approximation.

\section{DISCUSSION AND CONCLUSION}

We have considered collective effects in $\nu \bar{\nu}$ synchrotron radiation from an ultrarelativistic electron gas, under conditions such the electron motion is semiclassical. These conditions are satisfied by most astrophysical scenarios which are of interest for this process (neutron star crust, accretion disks and so on). We apply a calculation method which explicitly makes use of this fact, although it takes into account the interaction among particles in a quantum way. In order to test this method, we evaluated the single-particle synchrotron radiation in magnetized vacuum and compared it with previous results.

In the case of a degenerate electron gas, we performed our calculations assuming

$$
\frac{m T}{E_{F}^{2}} B_{0} \ll B \ll \frac{E_{F}^{2}}{m^{2}} B_{0}
$$

which typically hold in neutron star matter.

We discussed these collective effects for the process under consideration, which can be understood as a cloud of virtual particles and holes, which follows the radiating electron and also produce some flux of neutrino pairs going in the same direction as the $\nu \bar{\nu}$ radiation of the initial electron. They lead to an essential enhancement (almost three times) of the vector weak current contribution to neutrino pair emissivity. We have evaluated them focusing on the case 


$$
\frac{e B}{T E_{F}} \ll 1
$$

On one hand, this means that the temperature is high enough for many Landau levels to be occupied above the Fermi energy. On the other hand, the same condition means that the radiated frequency $(\omega \sim T)$ is much larger than the electron gyrofrequency and so, one can neglect the contribution of the external magnetic field to the medium polarization tensor. As it is obvious, if condition (88) is not satisfied, then synchrotron radiation of neutrino pairs will be exponentially reduced by the small population of excited Landau levels.

\section{ACKNOWLEDGMENTS}

This work has been partially supported by Spanish DGICYT Grant PB94-0973 and CICYT AEN96-1718. L.B. L. would like to thank for partial support of this work by RFFR Grant 97-02-16501. 


\section{REFERENCES}

[1] J. D. Landstreet, Phys.Rev., 153, 1372, (1967)

[2] V. Canuto, H. Y. Chiu and C. K. Chou, Phys.Rev., D2, 281 (1970)

[3] D. G. Yakovlev and R. Tschaepe, Astron. Nach., 302, 167 (1981)

[4] A. Vidaurre, A. Pérez, H. Sivak, J. Bernabéu and J. Mª . Ibañez, ApJ, 448, 264 (1995)

[5] A. D. Kaminker, K. P. Livenfish, D. G. Yakovlev, Soviet. Astron. Lett., 17, 450 (1991)

[6] A. D. Kaminker, K. P. Livenfish, D. G. Yakovlev, P. Amsterdamski and P. Haensel, Phys. Rev., D46, 3256 (1992)

[7] A. D. Kaminker and D. G. Yakovlev, JETP, 76, 229 (1993)

[8] V. N. Baier and V. M. Katkov, JETP, 52, 1422 (1967).)

[9] V. B. Berestetskii, E. M. Lifshitz and L. P. Pitaevskii, Quantum Electrodynamics. Landau and Lifshitz Course of Theoretical Physics, Volume 4, 2nd Edition. (Pergamon Press, Oxford, 1982), page 376.

[10] V. N. Baier and V.M. Katkov, Sov. Phys. Doklady, 171, 313 (1966).

[11] A.V. Borisov, V.Ch. Zhukovskii and P.A. Eminov, Ivuz. Fizika 3, 110 (1978).

[12] I. M. Ternov, V. N. Rodionov and A. I. Studenikin, Sov. J. Nucl. Phys. 37, 755 (1983).

[13] A. I. Nikishov and V. I. Ritus, JETP 46, 776 (1964)

[14] J. B. Adams, M. A. Ruderman and C.-H. Woo, Phys.Rev.,129, 1383 (1963)

[15] E. Braaten and D. Segel, Phys.Rev., D48, 1478 (1993) 


\section{FIGURES}

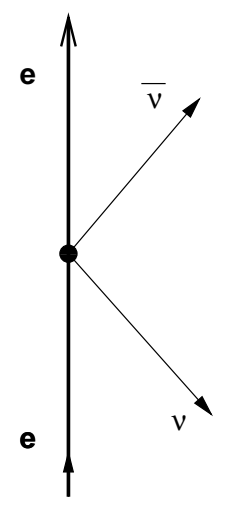

(1)

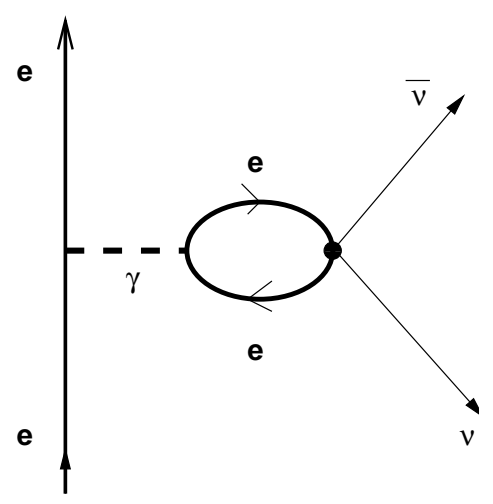

(2)

FIG. 1. Diagramms contributing to the matrix element of neutrino synchrotron radiation in a dense electron plasma. The low-energy weak interaction is shown as a filled cicle. We assume thick lines correspond to electrons in the external magnetic field. The thick dashed line in the second diagram represents the many-loop photon propagator in the medium.

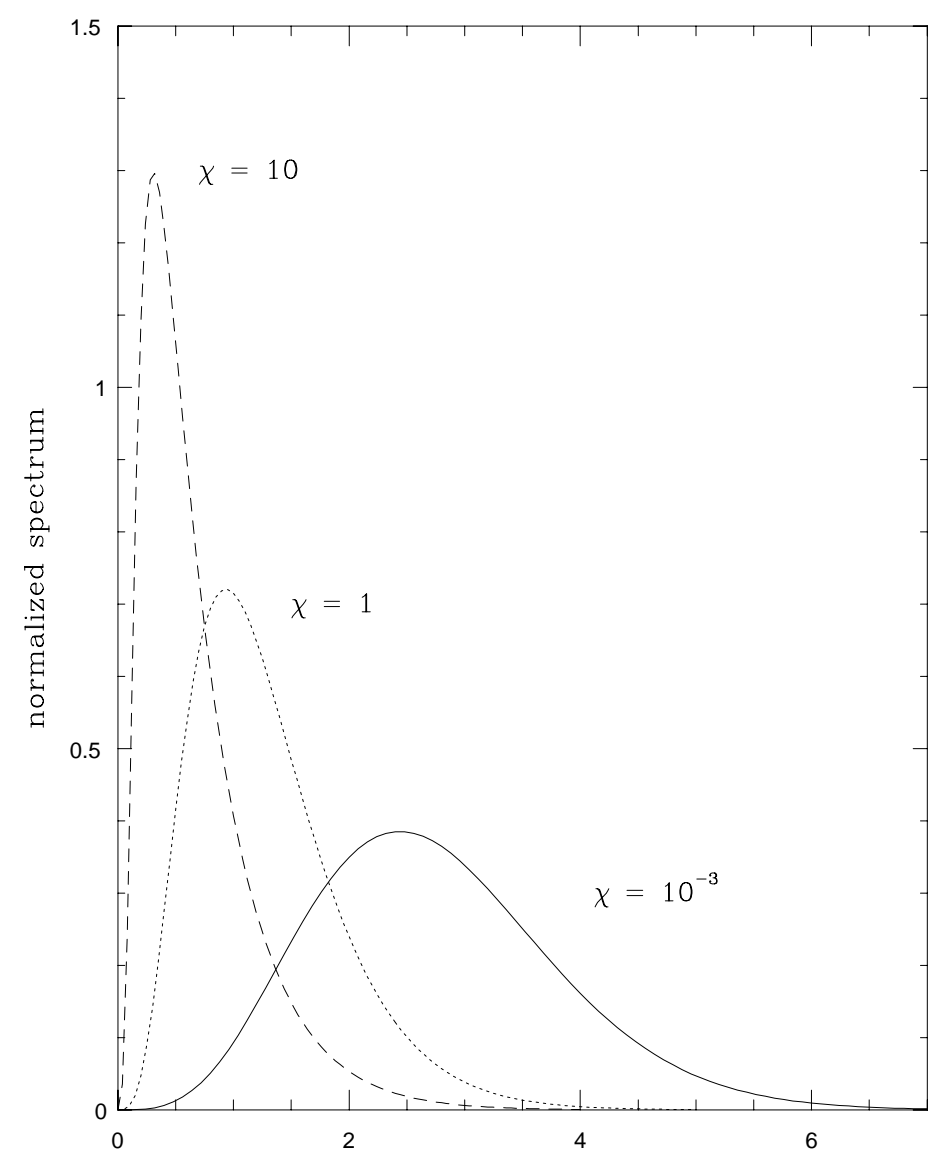

FIG. 2. Normalized neutrino pair energy spestrum as a function of the variable $s$, for three values of the parameter $\chi$, as labeled in the picture. 